\title{
Calculation of non-inferiority margin in pharmacoepidemiology: Simple methods for clinicians
}

\author{
Kourosh Zali
}

Arya Research Institute, Isfahan, Iran

*. Corresponding author: Kourosh Zali, Arya Research Institute, Iran, E-mail:

k.zali.arya.res.inst@gmail.com.

Cite this article: Zali K. Calculation of non-inferiority margin in pharmacoepidemiology: Simple methods for clinicians. Int J Epidemiol Health Sci 2021;2(6): e11. Doi: 10.51757/IJEHS.2.6.2021.243816.

\begin{abstract}
The presence of a placebo arm is certainly a substantial element in randomized control trials (RCTs) because the effectiveness or efficacy of a new therapy can be evaluated through a direct comparison between the test treatment and the placebo arm. Conducting a placebo controlled RCT is often very difficult or even impossible. To solve this problem, the availability of standard treatments and ethical concerns have led scientists to consider an active or positive control treatment as a comparator to assess the treatment effect without a placebo arm. Such an assessment is often made under a so-called "non-inferiority trial" (NIT) design. Non-inferiority margin (NIM) is the most important part of an NIT. Because there is no well-established method to determine the NIM, it is very important that this margin be pre-specified and the criteria for how it was established well defined prior to conducting the study. All methods of determination of NIM rely upon subjective judgment with unverifiable assumptions. This article demonstrates six simple methods to calculate NIM.
\end{abstract}

Keywords: Non-inferiority trial, Non-inferiority margin, Pharmacoepidemiology, Clinicians

After publication of my article about "non-inferiority trials (NITs) and non-inferiority margin (NIM)" in 2020 (1), I have received many emails about the simpler methods to calculate NIM. It is worth noting that NIM is based on the proportion of the therapeutic effect of the active control that should be retained (2). Generally, NIM is required to be smaller than or no greater than the effect of the selected active control under the NIT setting (3).

There is no well-established method to determine the NIM. Therefore, it should be pre-specified and well defined prior to conducting the study (4). All the methods for margin determination rely upon subjective judgment and unverifiable assumptions (5). Suppose that we consider "lack of equal or inferior efficacy in comparator arm" and "absolute difference between test arm and comparator arm" equals to $5 \%$. With these assumptions, there would be the following simple methods to calculate NIM:

1- Classic method (6):

If the suppression rate for the reference drug is greater than $95 \%$, then a difference in suppression rate within $5 \%$ is not considered a clinically important difference. If the suppression rate is between $80 \%$ and $90 \%$, then a NIM of $15 \%$ should be chosen.

2- Between 5\% and 10\% (7):

Usually, 50 to $75 \%$ is accepted as the fraction of the estimated control effect to be preserved compared to the placebo. To preserve $50 \%$, NIM must be equal to $10 \%$ and, to preserve $75 \%$, NIM must be equal to $5 \%$. At this point, clinical considerations may add in decisionmaking and the establishment of a final value.

3- Between $10 \%$ and $20 \%(7)$ :

NIM may be determined as a percentage of the control effect estimated for the current study, usually between 10 and $20 \%$. Its definition must, however, consider the therapeutic field and the magnitude of the control group effect; for example, for more 
conservative margins, $10 \%$ and $20 \%$ when the anticipated effect is inferior to $80 \%$.

4- $50 \%$ rule (7):

The value of NIM must be inferior (preferentially 50\%) to the inferior limit of the $95 \%$ CI obtained from historical data that compares control treatment and placebo. Suppose that the difference of $5 \%$ between proportions has been obtained from a sample of 400 patients per group and the $95 \% \mathrm{CI}$ is $2.86-7.14 \%$. Taking the half of the inferior limit $(2.86 \%)$, the value suggested to NIM is $1.43 \%$.

5- The fixed-margin or 95-95 method (8):

For example, if the entire effect of the active control relative to placebo is 0.05 with the sample size of 400 , then $95 \%$ CI of 0.05 would be 0.0286-0.0714.

The lower limit of $95 \%$ CI: 0.0286 should be considered.

Then, $50 \%$ or $75 \%$ of the lower limit of $95 \%$ $\mathrm{CI}$ is the largest loss of effect (inferiority):

$\mathrm{NIM}=0.50 * 0.0286=0.0143$

$\mathrm{NIM}=0.75 * 0.0286=0.0214$

6- The pooled estimate of event rate (5):

Suppose that the pooled estimate of event rate would be 0.05 .

If it is clinically decided that $75 \%$ of the pooled estimate must be preserved by the new drug to demonstrate non-inferiority, then: $\mathrm{NIM}=(1-0.75) * 0.05=0.0125$

If it is clinically decided that $50 \%$ of the pooled estimate must be preserved by the new drug to demonstrate non-inferiority, then: $\mathrm{NIM}=(1-0.50) * 0.05=0.025$

\section{References}

1. Zali, K. Non-inferiority trials and noninferiority margin: an overview Int $\mathrm{J}$ Epidemiol Health Sci. 2020;1(4):e04.

2. Rothmann, M.D., Tsou, H.H. On noninferiority analysis based on delta-method confidence intervals. J Biopharm Stat. 2003;13(3):565-83.

3. Hung, H., Wang, S. Statistical Considerations for Noninferiority Trial Designs Without Placebo Statistics Biopharmaceutical Res. 2013;5(3):239-47.

4. Brown, T.M. Design and interpretation of noninferiority studies: A clinician's perspective. J Nucl Cardiol. 2017;24(6):1994-7.
5. Althunian, T.A., de Boer, A., Groenwold, R.H.H., Klungel, O.H. Defining the noninferiority margin and analysing noninferiority: An overview. $\mathrm{Br} \mathrm{J}$ Clin Pharmacol. 2017;83(8):1636-42.

6. Chow, S., Song, F. On Selection of Margin in Non-Inferiority Trials. J Biom Biostat. 2016;7:301.

7. Pinto, V. Non-inferiority clinical trials: concepts and issues. J Vasc Bras. 2010;9(3):145-51.

8. Schumi, J., Wittes, J.T. Through the looking glass: understanding non-inferiority. Trials. 2011;12:106. 